\title{
An Innovative Method to Enhance Resolution and Contrast for Satellite Images Using Discrete Wavelet Transform
}

\author{
Ashwini R.Karande. ${ }^{1}$, A.L.Renke. ${ }^{2}$ \\ ${ }^{I}$ (Department of Electronics, K.I.T. 's College of Engineering/ Shivaji University, Maharashtra, India) \\ ${ }^{2}$ (Department of Electronics, K.I.T.'s College of Engineering/ Shivaji University, Maharashtra, India)
}

\begin{abstract}
Digital images have one major issue which is their resolution. As these images are being used in various fields of research where the visual appearance of an image plays a vital role IE is very important. In this paper, a method of resolution enhancement of a satellite image is proposed as satellite images play significant role in many applications such as geosciences studies, astronomy and geographical information. This proposed resolution enhancement method is based on the interpolation of the input image and high frequency components of input image which undergoes discrete wavelet transform (DWT). DWT is used to decompose the low resolution input image into different frequency sub-bands. After that all the sub-bands obtained by DWT undergoes interpolation. Difference image obtained by subtracting interpolated low frequency sub-band from low resolution input image is added to interpolated other three high frequency sub-bands. Finally all these images are combined to get a new image with enhanced resolution with the help of inverse DWT(IDWT). Estimating high frequency sub-bands helps in achieving sharper image. Proposed method has been tested on various satellite images. The quantitative (peak signal to noise ratio and root mean square error) and visual appearance of the output image shows that the proposed method is better than conventional and state of art image resolution enhancement techniques.
\end{abstract}

Keywords: Discrete wavelet transform(DWT), Dual Tree- Complex Wavelet Transform (DT-CWT),Image enhancement, inverse discrete wavelet transform(IDWT),Peak Signal to Noise Ratio (PSNR), Recursive Cycle Spinning (RCS),, Wavelet zero padding(WZP)

\section{Introduction}

Image enhancement is the process in which the quality of digital image is improved without having the knowledge of the source of degradation. IE is the main technique for improving the resolution and visual appearance of the image where the experts make decision on the basis of the image information. IE process is unable to increase the data or information content in the image, it only helps in improvement of the dynamic range and can highlight certain spacial features of interest in image. IE technique can be divided into two broad categories.

A) Spatial Domain Enhancement Method

The term spatial domain refers to the image plane i.e. the collection of pixels composing an image. Spatial domain techniques are the procedures that operate directly on these pixels. To achieve enhancement, pixel values are directly manipulated.

B) Frequency domain enhancement method

In this method, Fourier transform is used to transfer the image into frequency domain. Fourier transform of the image to be enhanced is taken to get that image I frequency domain. All the enhancement operations required are carried out on that Fourier transform and inverse transform is taken to produce the final enhanced image.

Satellite images can capture a lot of information, but they have issues with their resolution and also appeared as blurred. These all issues associated with satellite images make enhancement of those images more necessary as it can improve the visibility of images and all unwanted noise can be removed. IE helps in extracting useful information from satellite images. These satellite images are used for weather forecast, crop production forecast, military intelligence and metrology, disaster mitigation planning and recovery and many more. So the enhancement of these images is very much necessary.

Resolution enhancement techniques have became very important in many image and video processing applications. High resolution image is the key requirement in multiple images processing applications, for perfect and accurate processing and analysis of the image. There are two most common reasons which explains the need of the high resolution image. They are-

I. Pictorial representation of high resolution image has improved as compared to the low resolution image. Improved pictorial representation helps for human interpretation and machine vision.

II. For image analysis, more information is required. In case of satellite images it is must. High resolution image contain the more amount of information as compared to the low resolution input image. 
Therefore, Image Enhancement is an important and critical application in field of image processing when it comes to enhancing satellite image at a resolution higher than that at which image is available. The Enhanced satellite images make diagnostic details more obvious.

Wavelets play an important role in the image processing field. A wavelet is a small wave. It is a mathematical function which is able to divide a given function or continuous- time signal into different scale component. A signal is decomposed in its basic functions by using wavelet transform . 2-D wavelet decomposition of an image is done with the help of 1-D wavelet transform(DWT). it is applied along the rows first and then the result obtained are decomposed along the columns. Four decomposed frequency sub-bands come as a result of this operation which are low-low(LL),low-high(LH),high-low(HL), and high-high(HH). full frequency spectrum of the original input image is covered by the frequency components of these sub-bands. Fig 1. shows the block diagram of this whole operation. Fig 2. shows different frequency sub-bands of a satellite image generated after undergoing DWT.

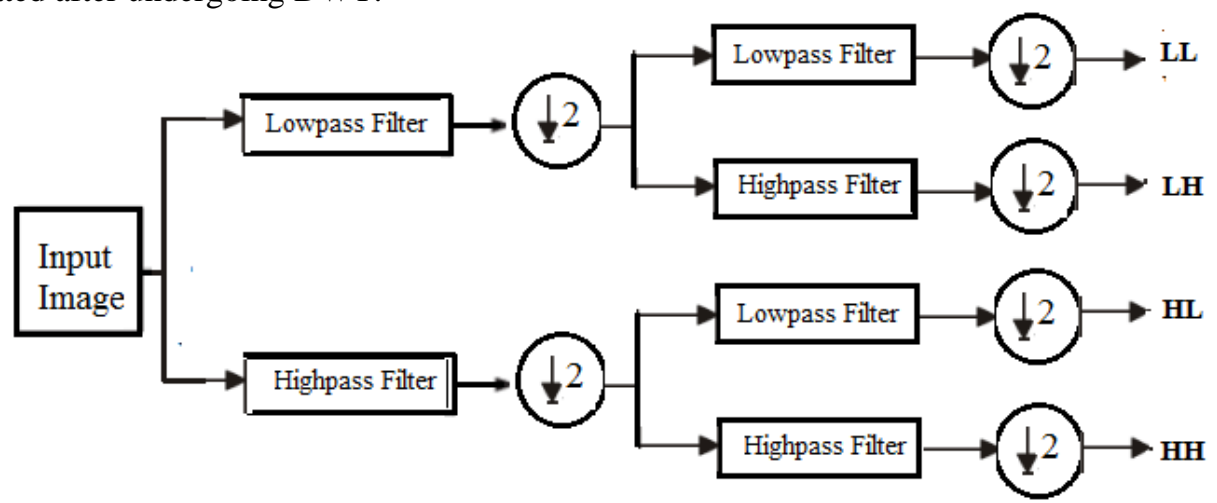

Fig 1: block diagram of DWT filter banks of level-1
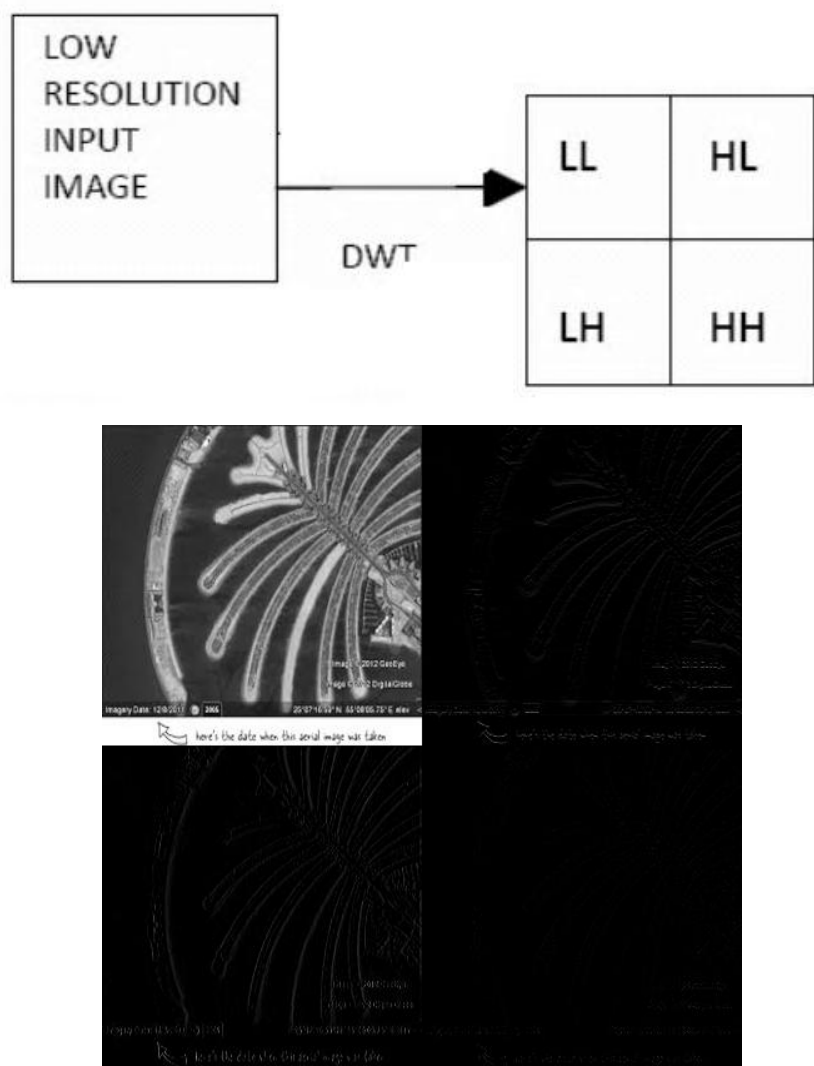

Fig 2: LL,LH,HL and HH sub-band obtained by using DWT

Contrast enhancement can be defined as the slope of the function mapping input intensity to output intensity. If it is assumed that the range of input and output intensities are the same. Then no enhancement is involved in slope of 1 and increasingly higher enhancement is given by higher slopes. Thus to involve restricting the slope of the mapping function, the limitation of contrast enhancement can be taken. 
The mapping function $\mathrm{m}(\mathrm{i})$ is proportional to the cumulative histogram with histogram equalization. $m(i)=($ Display_Range $) *($ Cumulative_Histogram $(i) /$ Region_size $) .. . . .(1)($ courtesy[6])

Since the derivative of the cumulative histogram is the histogram, the slope of the mapping function at any input intensity,I.e., the contrast enhancement , is proportional to the height of the histogram the intensity:

$$
\frac{d m}{d i}=(\text { Display_Range/Re gion_Size })^{*} \text { histogram }(i) \ldots \ldots . . .(2)(\text { courtesy[6]) }
$$

Therefore, limiting the slope of the mapping function is almost similar to clipping the height of the histogram. Retinex is an image enhancement algorithm that improves the brightness, contrast and sharpness of an image. Retinex stands for Retina+ cortex. Retinex improves visual rendering of an image when lightning conditions are not so good. The Multi Scale Retinex with Color Restoration (MSRCR) algorithm is the root of the Retinex filter.The multi scale retinex method is represented by the equation shown below.

$$
R_{i}\left(x_{1}, x_{2}\right)=\sum_{k=1}^{k} W_{k}\left\{\log I_{i}\left(x_{1}, x_{2}\right)-\log \left[F_{K}\left(x_{1}, x_{2}\right) * I_{i}\left(x_{1}, x_{2}\right)\right]\right\} \quad i=1,2, \ldots \ldots \mathrm{N}
$$

..... (3) (courtesy [7])

where index $i$ references the $i$ 'th spectral band, $(x 1, x 2)$ is the pixel location in Cartesian coordinates, and $*$ is the convolution operator. $N$ is the number of spectral bands $N 51$ for grayscale images, and $N 53, i \mathrm{P} R, G, B$ for typical color images. $I$ is the input image and $R$ is the output of the MSR process. $F k$ is the $k$ 'th $\sim$ Gaussian! surround function, $W k$ is the weight associated with $F k$, and $K$ is the number of surround functions, or scales.

The $F k$ are given as:

$$
F_{k}\left(x_{1}, x_{2}\right)=k \quad \exp \left[-\left(x_{1}^{2}+x_{2}^{2}\right) / \sigma_{k}^{2}\right.
$$

...(4) (courtesy [7])

where, $\sigma_{k}$ are the standard deviations of the Gaussian surrounds. The magnitude of $\sigma_{k}$ controls the extent of the surround: smaller values of $\sigma_{k}$ result in narrower surrounds. The MSR output is normalized by k. The MSR reduces to the single scale retinex, when $K=1$, with the additional constraint that $\mathrm{W} 1=1$.

In this paper, A satellite image resolution enhancement technique based on the interpolation of the high-frequency sub-band images obtained by Discrete Wavelet Transform (DWT) and the input image is proposed. All the sub-band images obtained are combined with the help of inverse DWT (IDWT) to generate final high resolution output image. Intermediate stage of estimating high frequency sub-bands by using difference image which is obtained by subtracting interpolated LL sub-band from input image gives sharper output image. The proposed technique has been compared with the standard interpolation technique. The visual and quantitative results are given in result and discussion section. In all steps of proposed satellite image resolution enhancement technique, Haar wavelet transform as mother wavelet function and bi-cubic interpolation technique have been used.

The paper is organized as follows. Section II gives an overview on the existing image resolution enhancement techniques. Section III introduces the proposed resolution enhancement technique based on Discrete Wavelet Transform. Section IV discusses the qualitative and quantitative results of the proposed method. Conclusions are given in the final section.

\section{WAVELET BASED IMAGE RESOLUTION ENHANCEMENT}

Many methods have been used for satellite image resolution enhancement. Various authors have proposed many new algorithms on image resolution enhancement using wavelets.Temizel and T. Vlachos (2005)[8] in their paper, Recursive Cycle Spinning (RCS) method was implemented to avoid the artifacts. With this method is able to get the better visual appearing images and Peak Signal to Noise Ratio (PSNR) value compared with the methods like wavelet image de-noising using the Recursive Cycle Spinning. Artifact is a classic signal interpolation problem and conventional approaches such as zero order interpolation (sample-andhold) cause severe pixelation impairments while bilinear and spline interpolation invariably results in undesirable levels of smoothing across salient edges. There is one common feature in all these algorithms is the assumption that the low resolution (LR) image to be enhanced is obtained the down-sampled image of a highresolution (HR) image which has been subjected to a decimated wavelet transform. 
Hasan Demirel And Gholamreza Anbarjafari. (2010)[9] complex wavelet transform (CWT) based image processing technique is proposed in his paper. Using CWT of an image two complex valued low frequency sub band image, and six complex valued high frequency sub band images are formed. DT-CWT produces dissimilar sub band images using original image. Sub-band images containing high frequency undergoes interpolation along with low frequency image. These real valued image are used for the IDT-CWT operation. This also improves the quality and PSNR of the input image and get super resolved output image.

Image super resolution based on interpolation of wavelet domain high frequency sub-bands and the spatial domain input image [10] This is one of the wavelet based image resolution enhancement method.The image undergoes interpolation operation while being super-resolved. This operation results in smoothing. Because of which Loss of the high frequency components of that image(I.e. the edges) occur. If the edges are preserved, an increased quality super resolved image can be obtained.In [10] work by Hasan Demirel and Gholamreza Anbarjafari, DWT has been employed. It is used to preserve the high-frequency components of the image. With the DWT an image is decomposed into different sub-band images; namely, low-low (LL), low-high (LH), highlow (HL), and high-high (HH). These sub-band images contain the high-frequency components of the input image. The interpolation is applied to high-frequency sub-band images as well as the the input image. The interpolation method employed is the same for all.The final high-resolution output image is produced by taking IDWT of the interpolated sub-band images and the input image .

Image resolution enhancement method using SWT and DWT [11]. In this technique use of DWT is dine to decompose an image into different frequency sub-band images, and then the interpolation process is applied to high frequency sub-band images. The high frequency sub-bands achieved by SWT of the input image are used to correct the interpolated high frequency sub-band coefficients. An original input low resolution image is interpolated. Interpolation factor used for this is half of the interpolation factor used to interpolate the high frequency sub-bands. After that all the sub-band images combined using IDWT to generate an image having super resolution. The output image contains sharper edges than the interpolated image obtained by interpolation of the input image directly. This happens due to the fact that, the interpolation of isolated high frequency components in high frequency sub-bands and the corrections are used which are obtained by adding high frequency sub-bands of SWT of the input image. It helps in will preserving more high frequency components after the interpolation than interpolating input image directly.

\section{Dwt Based Resolution And Contrast Enhancement Method}

As discussed earlier, resolution of an image is important factor in satellite imaging. Because of this, the resolution enhancement of image is very important as it results in visually better enhanced images than the original low resolution image.

The proposed DWT based interpolation technique consists of steps as follows:

1. Proposed method starts by decomposing the low resolution input image in to different frequency sub-bands namely, LL, LH, HL and HH.

2. All the four frequency coefficients are then interpolated with the help of bi-cubic interpolation.

3. Find the difference between input image and the interpolated low frequency sub-band image.Add this difference to other interpolated high frequency sub-bands for correct estimation of the high frequency component.

4. At last, Inverse discrete transform is applied to get the high resolution enhanced image. It performs the reverse operation of DWT. Inverse DWT (I-DWT) combines all the high frequency sub-band images and the low resolution input image to obtain a final resolution enhanced image.

DWT is used in order to preserve the high-frequency components of the image. DWT separates the image into different sub-band images, namely, LL, LH, HL, and HH. A high-frequency sub-band contains the high frequency component of the image. DWT is employed to preserve the high frequency components. The first sub-band (the LL sub-band) will contain low pass information, which is essentially a low-resolution version of the image. Sub-band HL will contain low pass information vertically and high pass information horizontally, and sub-band LH will contain low pass information horizontally and high pass information vertically. Finally, sub-band $\mathrm{HH}$ will contain high pass information in both directions. Low frequency Sub-band images are are obtained by low pass filtering the low resolution of the original image. These low frequency sub-band images contains less information as compared to original image. Therefore, instead of using low-frequency sub-band images, input image is used through the interpolation process. Hence, the interpolation factor used to interpolate the input low-resolution image is the half of the interpolation factor $(\alpha / 2)$ used to interpolate the high-frequency sub-bands $(\alpha)$, as shown in Fig. 3. In order to obtain a sharper enhanced image (i.e. to preserve more edge information) an intermediate stage in high frequency sub-band interpolation process is proposed. Bicubic interpolation produces noticeably sharper images than other interpolation methods. 
As shown in Fig. 3, the interpolated LL image with factor 2 and the low-resolution input satellite image are highly correlated. The difference between the low-resolution input image and the interpolated LL frequency sub-band are in their high-frequency components. Hence, this difference image obtained is used at the intermediate process which will correct the high-frequency components which are estimated. This estimation is obtained by interpolating the high-frequency sub-bands by interpolation factor 2 and after that including the difference image (which is high-frequency components on low-resolution input image) into the estimated highfrequency images, follows another interpolation with factor $\alpha / 2$ which is required in order to get the required size for Inverse DWT process. The intermediate process of addition of the difference image which is containing high-frequency components, results in significantly sharper and clearer output image. This sharpness is improved by the fact that, the interpolation carried on isolated high-frequency components in $\mathrm{HH}$, HL, and LH will results in preserving more high-frequency components than obtained by directly interpolating the lowresolution image.

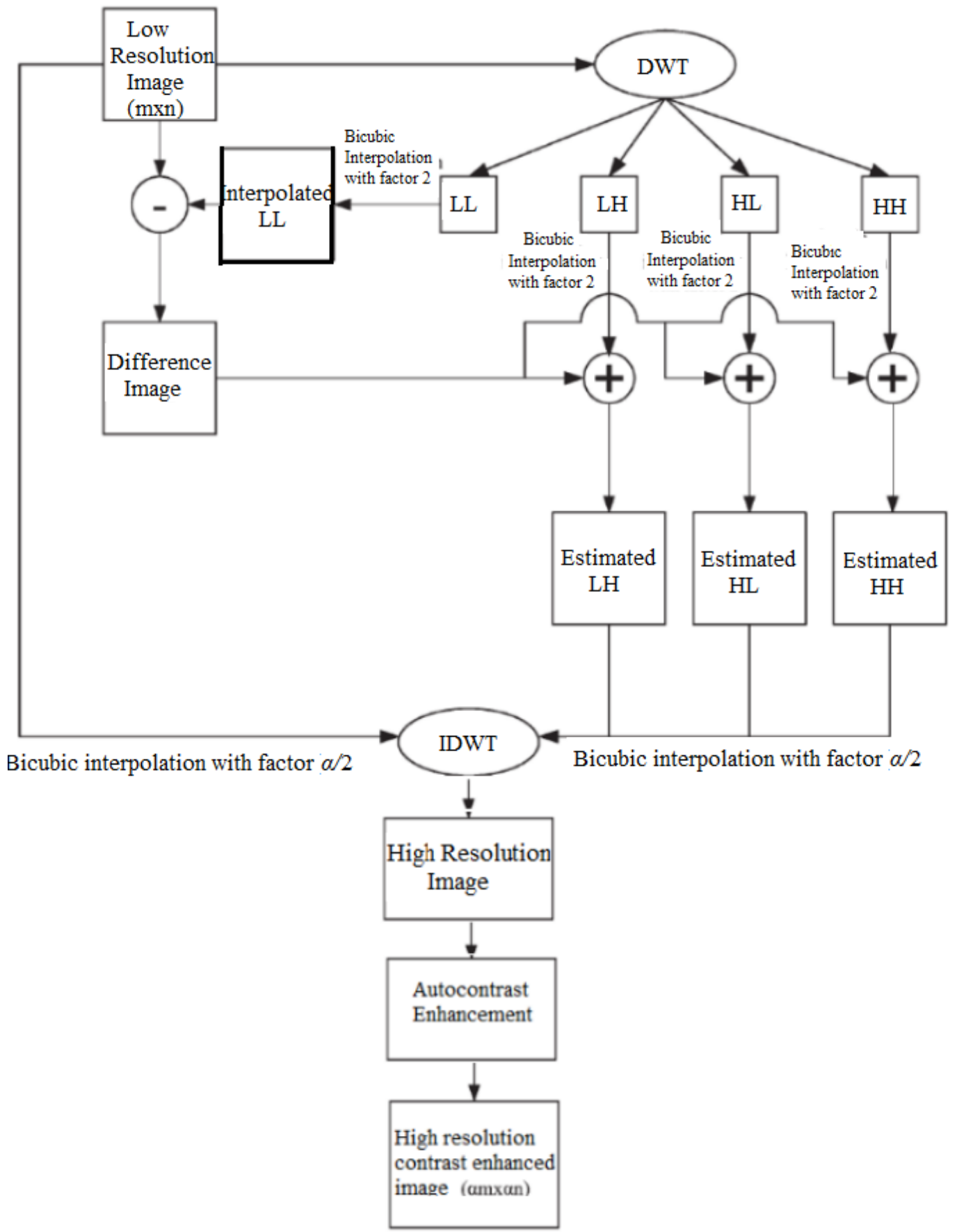

Fig.3: Block diagram of the proposed DWT based resolution and contrast enhancement method 
(1)

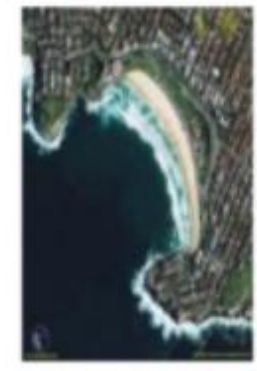

(2)

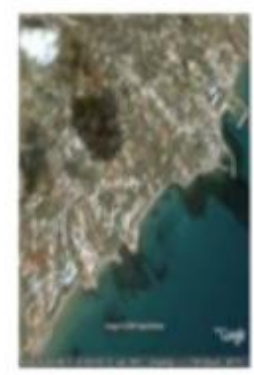

(a)
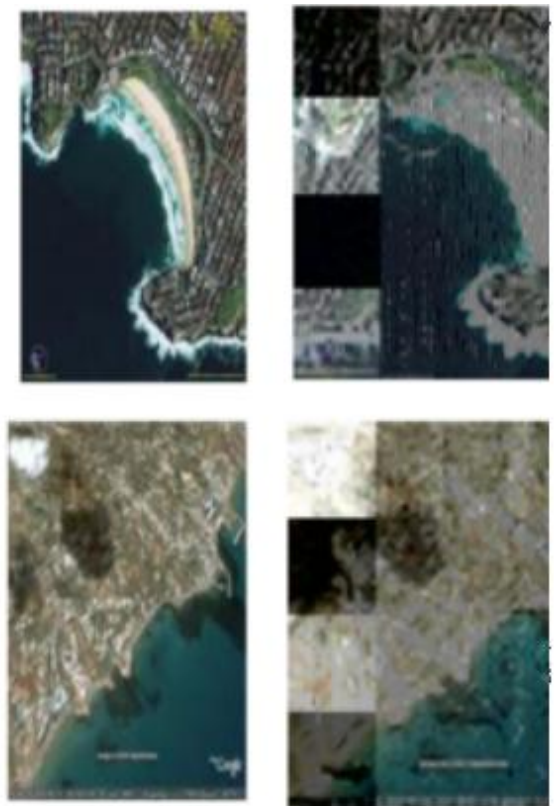

(b)

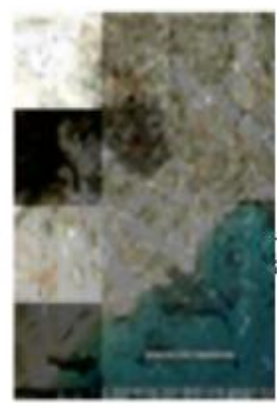

(c)

Figure 4: (a) Input image (b)Resolution enhanced image (output of IDWT) (c) Output of Bicubic interpolation

(all the figures are taken from the Google search engine services, directly saved to the hard disk, processed with the proposed algorithm and the quantitative results are tabulated)

Figs. 4 (a)-(c) show the difference between the low resolution images with the intermediate resolution enhanced image (I.e. output of IDWT stage) by using the proposed resolution enhancement technique, the image obtained by using bicubic interpolation technique directly, respectively. Figs. 4 shows that more high-frequency components have been preserved in the proposed technique.

Output of IDWT is also a High Resolution image of size $\alpha \mathrm{M} x \alpha \mathrm{N}$, but it has less contrast information. Further the output of IDWT is given as input to the auto contrast enhancement algorithm. The final output we get is 'High Resolution Contrast Enhanced Image'.

\section{Results And Discussions}

The proposed technique has been tested on several different satellite images. Fig 5 shows the superiority of the proposed method over the conventional and state of art technique from visual point of view. In this fig.5 The resolution enhanced image by using DWT, resolution and contrast enhanced image using proposed method, and the original input image are shown. From those images it is clear that the resultant image, enhanced by using proposed technique, is sharper than the input image. The figure 6 indicates the screen shot of the output of implemented code.
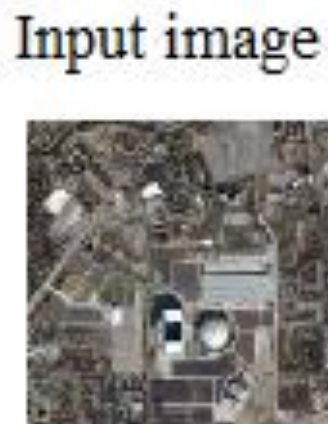

HR Image ,

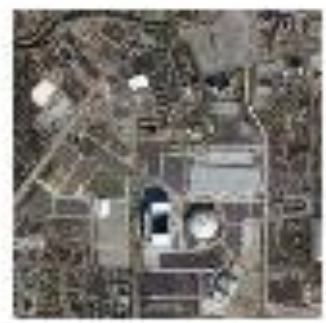

$\mathrm{HR}+$ Auto-contrast

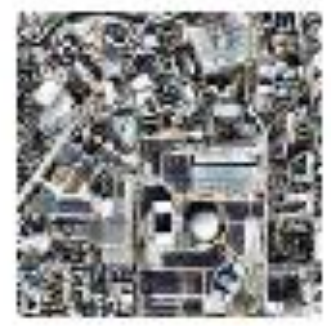

Fig.5 Analysis between (a) Input image, (b) High Resolution Image, (c) High Resolution High Contrast Image 


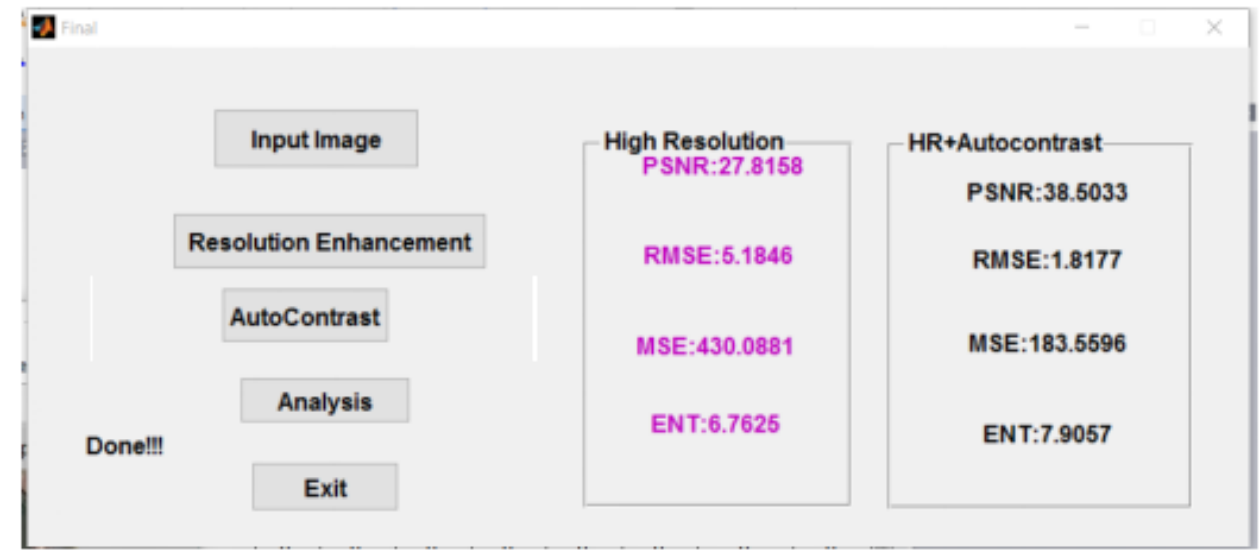

Fig.6 Screen shot of the output of implemented code

\section{Performance Analysis:-}

Along with the visual comparison, the quantitative comparisions also confirms the superiority of the proposed method. 10 satellite images were taken from the Google search engine and the quantitative results were calculated after applying the implemented code.

The superiority of the proposed method will get confirmed with quantitative comparisons in addition with visual comparison.Peak signal-to-noise ratio (PSNR) and root mean square error (RMSE) are implemented in order to obtain some quantitative results for comparison. PSNR can be obtained by using the following formula:

$$
P S N R=10 \log _{10}\left(\frac{R^{2}}{M S E}\right) \ldots \ldots \ldots \ldots \ldots(5)(\text { courtesy [16]) }
$$

Where

$\mathrm{R}$ is the maximum fluctuation in the input image and MSE is representing the Mean Square Error between the given input image $\boldsymbol{I}_{\text {in }}$ and the original image $\boldsymbol{I}_{\text {org }}$ which can be obtained by the following:

$$
M S E=\frac{\sum_{i, j}\left(I_{\text {in }}(i, j)-I_{\text {org }}(i, j)\right)^{2}}{M X N}
$$

Where, $\mathrm{M}$ and $\mathrm{N}$ are the size of the images.

Clearly, RMSE is the square root of Mean Square Error (MSE); hence it can be calculated by the following:

$$
R M S E=\sqrt{M S E}=\sqrt{\frac{\sum_{i, j}\left(I_{\text {in }}(i, j)-I_{\text {org }}(i, j)\right)^{2}}{M X N}} \ldots \ldots \ldots . .(7)(\text { courtesy [16]) }
$$

TABLE 1: PSNR Analysis

\begin{tabular}{|c|c|c|c|c|c|c|c|c|c|c|}
\hline & \multicolumn{10}{|c|}{ RMSE } \\
\hline Method \Image & Fig.1 & Fig.2 & Fig.3 & Fig.4 & Fig.5 & Fig.6 & Fig.7 & Fig.8 & Fig.9 & Fig.10 \\
\hline
\end{tabular}

\begin{tabular}{|c|c|c|c|c|c|c|c|c|c|c|}
\hline & \multicolumn{9}{|c|}{ PSNR (dB) } \\
\hline Method $\backslash$ Image & Fig.1 & Fig.2 & Fig.3 & Fig.4 & Fig.5 & Fig.6 & Fig.7 & Fig.8 & Fig.9 & Fig.10 \\
\hline $\begin{array}{c}\text { DWT based method output (High } \\
\text { Resolution Image) }\end{array}$ & 27.81 & 29.05 & 29.05 & 28.99 & 29.44 & 27.95 & 28.97 & 28.74 & 29.71 & 28.39 \\
\hline HR+Auto contrast Enhancement & 38.50 & 34.78 & 30.90 & 32.16 & 43.41 & 31.02 & 33.16 & 37.30 & 41.15 & 35.78 \\
\hline
\end{tabular}

TABLE 2: RMSE Analysis 
An Innovative Method To Enhance Resolution And Contrast For Satellite Images Using Discrete ..

TABLE 3: MSE Analysis

\begin{tabular}{|c|c|c|c|c|c|c|c|c|c|c|}
\hline & \multicolumn{10}{|c|}{ MSE } \\
\hline Method \Image & Fig.1 & Fig.2 & Fig.3 & Fig.4 & Fig.5 & Fig.6 & Fig.7 & Fig.8 & Fig.9 & Fig.10 \\
\hline $\begin{array}{l}\text { DWT based method output } \\
\text { (High Resolution Image) }\end{array}$ & 430 & 323 & 323 & 328.06 & 295.76 & 416.8 & 328 & 347.30 & 277.90 & 376.44 \\
\hline $\begin{array}{l}\text { HR+Auto contrast } \\
\text { Enhancement }\end{array}$ & 183 & 431 & 211 & 157.88 & 59.26 & 205.43 & 125 & 242.01 & 99.76 & 343.55 \\
\hline
\end{tabular}

TABLE 4: Entropy Analysis

\begin{tabular}{|c|c|c|c|c|c|c|c|c|c|c|}
\hline & \multicolumn{10}{|c|}{ ENTROPY } \\
\hline Method \Image & Fig.1 & Fig.2 & Fig.3 & Fig.4 & Fig.5 & Fig.6 & Fig.7 & Fig.8 & Fig.9 & Fig.10 \\
\hline $\begin{array}{c}\text { DWT based method } \\
\text { output (High Resolution } \\
\text { Image) }\end{array}$ & 6.7625 & 6.4583 & 6.0683 & 5.8754 & 6.4161 & 6.123 & 6.2511 & 6.632 & 6.7567 & 6.6457 \\
\hline $\begin{array}{l}\text { HR+Auto contrast } \\
\text { Enhancement }\end{array}$ & 7.9057 & 7.4603 & 7.7288 & 7.545 & 7.5909 & 7.92 & 7.7992 & 7.7268 & 7.646 & 7.9234 \\
\hline
\end{tabular}

Table 1 indicates the PSNR analysis done on 10 images. The PSNR of High resolution is up to acceptable level. The additional enhancement in terms of contrast gives better results after high resolution. Table 2 and Table 3, indicate the RMSE and MSE analysis done on 10 images. The RMSE and MSE of High resolution is up to acceptable level. The additional enhancement in terms of contrast gives better results after high resolution.

In order to show the improvement obtained by the proposed satellite image enhancement from information content point of view, Table 4 indicates the entropy analysis done on 10 images. The entropy of High resolution is up to acceptable level which is within the range 5 to8. The additional enhancement in terms of contrast gives better results after high resolution by preserving entropy.

\section{Conclusion}

This paper has proposed a resolution and contrast enhancement technique based on the interpolation of high frequency sub-band images obtained by Discrete Wavelet Transform(DWT) and the input image. The proposed enhancement technique has been tested on different satellite images taken from Google search engine. The proposed resolution and contrast enhancement technique outperforms. It fives superior resolution when compared to existing technique. The visual and quantitative results of the proposed method show the superiority of the proposed technique. The PSNR result obtained after high resolution are up to acceptable level. Further enhancement is done by using contrast enhancement. The output image from entire process provides good results.

\section{References}

[1] Ahmed M. Mahmood, and Jasni Mohmad Zain. “A study on the validation of histogram equalization as a contrast enhancement technique". In Advanced Computer Science Applications and Technologies (ACSAT),2012 International Conference on, pp.253256.IEEE,2012.

[2] Cao, Gang, Yao Zhao, Rongrong Ni, and Xuelong Li. "Contrast enhancement-based forensics in digital images." Information Forensics and Security, IEEE Transactions on 9, no. 3 (2014): 515-525.

[3] Cheng, H. D., and Yingtao Zhang. "Detecting of contrast over-enhancement." In Image Processing (ICIP), 2012 19th IEEE International Conference on, pp. 961-964. IEEE, 2012.

[4] Chen, Xiaoming, and Lili Lv. "A Compositive Contrast Enhancement Algorithm of IR Image." In Information Technology and Applications (ITA), 2013 International Conference on, pp. 58-62. IEEE, 2013.

[5] Reshmalakshmi, C., and M. Sasikumar. "Image contrast enhancement using fuzzy technique." In Circuits, Power and Computing Technologies (ICCPCT), 2013 International Conference on, pp. 861-865. IEEE, 2013.

[6] "Adaptive Histogram Equalization and Its Variation"

[7] Zia-ur Rahman,Daniel J. Jobson,Glenn A. Woodell " Retinex processing for automatic image enhancement"

[8] Temizel and T. Vlachos, "Wavelet domain image resolution enhancement using cycle-spinning," Electron. Lett., vol. 41, no. 3, pp. 119-121, Feb. 3, 2005.

[9] Hasan Demirel And Gholamreza Anbarjafari,"Satellite Image Resolution Enhancement Using Dual Tree-Complex Wavelet Transform" IEEE Trans. Geoscience And Remote Sensing Letters, Vol.7, No.1, January 2010,Pp123-126.

[10] Y. Piao, L. Shin, and H. W. Park, "Image resolution enhancement using inter-sub-band correlation in wavelet domain," in Proc. IEEE ICIP, 2007, vol. 1, pp. I-445-I-448.

[11] Hasan. Demirel and Gholamreza. Anbarjafari, -IMAGE Resolution Enhancement by Using Discrete and Stationary Wavelet Decomposition,\|IEEE IMAGE PROCESSING, VOL. 20, NO. 5, MAY 2011..

[12] Demirel, Hasan, CagriOzcinar, and Gholamreza Anbarjafari. "Satellite image contrast enhancement using discrete wavelet transform and singular value decomposition." Geoscience and Remote Sensing Letters, IEEE 7.2: pp.333-337, 2010.

[13] Maragatham, G., S. MdMansoor Roomi, and T. Manoj Prabu. "Contrast enhancement by object based Histogram Equalization." Information and Communication Technologies (WICT), 2011 World Congress on. IEEE, 2011.

[14] Arya P Unni, "Satellite Image Enhancement Using 2D Level DWT", International Journal of Engineering Research \& Technology (IJERT), ISSN: 2278-0181, Vol. 3 Issue 3, March 2014.

[15] Turgay Celik And Huseyin Kusetogullari," Self-Sampled Image Resolution Enhancement Using Dual-Tree Complex Wavelet Transform" In European Signal Processing Conference, Glasgow, Scotland, 2009. 
[16] Hasan Demirel And Gholamreza Anbarjafari,'Discrete Wavelet Transform-Based Satellite Image Resolution Enhancement” IEEE Trans. Geoscience And Remote Sensing Letters, Vol.7, No.5,May 2011.

[17] Ke, Wei-Ming, Chih-Rung Chen, and Ching-Te Chiu. "BiTA/SWCE: Image enhancement with bilateral tone adjustment and saliency weighted contrast enhancement." Circuits and Systems for Video Technology, IEEE Transactions on 21.3: pp.360-364, 2010.

[18] Chauhan, Ritu, and Sarita Singh Bhadoria. "An improved image contrast enhancement based on histogram equalization and brightness preserving weight clustering histogram equalization." Communication Systems and Network Technologies (CSNT), 2011 International Conference on. IEEE, 2011.

[19] Ghimire, Deepak, and Joonwhoan Lee. "Nonlinear transfer function-based local approach for color image enhancement." Consumer Electronics, IEEE Transactions on 57.2: pp.858-865, 2011

[20] Josephus, ChelsySapna, and S. Remya. "Multilayered Contrast Limited Adaptive Histogram Equalization Using Frost Filter." Recent Advances in Intelligent Computational Systems (RAICS), IEEE, 2011.

[21] Lasmika, K. Raveendra, "Improving Quality of Satellite Image by Wavelet Transforming \& Morphological Filtering", International Journal of Innovative Research in Science, Engineering and Technology, ISSN: 2319- 8753,Vol. 3, Issue 7, July 2014.

[22] E. Mohan, K.B. Jayarraman, U. Maheswaran, D. Sathiyaraj. G.Dhakshanamoorthi,"A novel aproach for satellite image resolution enhancement", International Journal Of Engineering And Advanced Technology (Ijeat) Issn:2249-8958, Volume-2, Issue-4, April 2013,Pp.112-114

[23] S.VENKATRAMANA, S. NARAYANA REDDY," A Novel Method To Improve Resolution Of Satellite Images Using DWT And Interpolation." International Journal Of Advanced Research In Electrical, Electronics And Instrumentation Engineering, Vol.3, Issue 1,January 2014,Pp.6530-6536

[24] Sundaram, M., K. Ramar, N. Arumugam, and G. Prabin. "Histogram based contrast enhancement for mammogram images." In Signal Processing, Communication, Computing and Networking Technologies (ICSCCN), 2011 International Conference on, pp. 842-846. IEEE, 2011

[25] Madhu S. Nair. And G. Raju "A fast and efficient color image enhancement method based on fuzzy logic and histogram". IEEE Trans Syst Man Cybern 1981;SMC-11(July (2013):494-501ss[6]

[26] X. Li and M. T. Orchard, "New edge-directed interpolation,” IEEE Trans. Image Process., vol. 10, no. 10, pp. 1521-1527, Oct.2001. 